\title{
PHILOSOPHIE
}

Gilbert Romeyer-Dherbey, Les Sophistes. Paris, P.U.F., 1985. 11,2 × 17,6, 128 p. ("Que sais-je ? ", $n^{\circ} 2223$ ).

La parution d'un "Que sais-je ? » consacré aux Sophistes est sans doute le signe que la réhabilitation est désormais irréversible de ces Indiens de la philosophie qui, depuis les débuts platoniciens d'un long Western théorique, avaient toujours tort. Certes les Sophistes ne sont pas les seuls penseurs antiques dont il ne nous reste que des fragments principalement conservés dans les citations et allusions des autres. Il en est de même pour les Présocratiques, les anciens Stoïciens... Mais dans le cas des Sophistes (du moins de ceux de la " première " sophistique, c'est-à-dire les contemporains de Socrate et Platon, les seuls dont il soit question ici) il ne s'agit pas seulement d'une perte de textes. La tradition qui les porte à notre connaissance est si malveillante à leur égard qu'il faudrait parler de " bibliocide ".

Cette " vulgarisation », au meilleur sens du terme, des Sophistes, prend la forme d'un hommage, non seulement parce que G. Romeyer-Dherbey est " du côté » des Sophistes, mais aussi parce que les « vulgarisateurs " géniaux que furent les Sophistes en auraient sans doute approuvé le projet. Hommage malaisé dans un cadre aussi restreint : rien d'aussi difficile à écrire que les petits livres quand ils ne portent pas sur de petits sujets. L'auteur a réussi dans son entreprise, en conjuguant une appréciation d'ensemble vigoureuse de la figure de chacun des Sophistes avec une connaissance impeccable des textes.

D'entrée de jeu, il pose le problème des " traits communs qui valent aux Sophistes une dénomination semblable » et le résout bien, c'est-à-dire de l'extérieur et rapidement. La tradition caractérise d'abord les Sophistes par des thèmes communs (par exemple le fameux thème des rapports entre la nature et la loi). Sans rejeter ce critère, G. Romeyer-Dherbey a raison de le faire passer au second plan : il évite ainsi un conflit entre l'extension et la compréhension

Revue de synthèse : IVe S. $\mathrm{N}^{\circ} 3$, juillet-septembre 1986. 
du concept de Sophiste (tous les Sophistes ne traitent pas de tel thème réputé " sophistique " et qui pourtant se retrouve chez des non-Sophistes). En deux pages, il montre fort bien que « la ressemblance qui relie les individualités distinctes [des Sophistes] est plutôt celle d'un moment historique et d'un statut social » (p. 4). Les Sophistes sont des professionnels du savoir, itinérants, rendus en quelque sorte nécessaires par le développement des institutions démocratiques. Un " mouvement " donc, et non pas une école.

L'auteur peut ensuite, en huit chapitres d'inégale longueur, dresser le portrait historique et théorique des huit grandes figures de la Sophistique ancienne. Protagoras et Gorgias sont, comme dans les autres ouvrages consacrés aux Sophistes, traités plus longuement que leurs « collègues ». Effet nécessaire d'une disparité dans la transmission des textes, et non, chez Romeyer-Dherbey, nous le verrons, trace d'une préférence pour ces deux Sophistes-là. Il faut souligner qu'à côté de l'exposé riche et précis des doctrines, il sait faire la part, indispensable quand on parle des Sophistes, de l'anecdote (par exemple p. 34 : les échanges acides entre Gorgias et Platon). G. Romeyer-Dherbey sait aussi donner une épaisseur historique à sa propre interprétation en la situant par rapport aux autres interprétations, proches ou lointaines de nous. Un modèle de ce genre d'analyse est fourni par l'interprétation que l'auteur propose de la fameuse doctrine protagoréenne de " l'homme mesure de toutes choses ". Remontant à Hegel, reprenant, mais en les critiquant, les thèses d'Untersteiner (I Sophisti, testimonianze e frammenti, Florence, 1949, 4 fascicules : la meilleure édition, et la meilleure traduction, disponible des textes concernant les Sophistes), l'auteur fait définitivement justice des interprétations relativiste et sceptique de la célèbre formule : "l'homme " c'est, non pas par " une confusion involontaire, mais [par] une fusion voulue ", à la fois, et tour à tour, l'homme individuel et l'homme universel, et « la vérité gît précisément dans le passage du premier au second sens : l'opinion personnelle se vérifie par son accord avec les opinions des autres » (p. 22).

Mais les autres Sophistes ne sont pas négligés, en des analyses qui, toujours, corrigent, précisent, nuancent. Ainsi Prodicos ne serait-il considéré comme pré-évhémériste que par illusion rétrospective ; ainsi Thrasymaque a-t-il été le plus mal traité par la parodie platonicienne, puisque Platon fait de ce démocrate conciliateur un partisan de la tyrannie en détournant de son sens la critique thrasymaquéenne de l'injustice de la loi. Mais, pour G. Romeyer-Dherbey, le " grand » Sophiste c'est Antiphon. « Ce dont on peut s'étonner [...], écrit-il, c'est l'acharnement mis par l'histoire à effacer la trace d'un penseur de cette trempe. De sa personne nous ne savons rien (ou à peine qu'il n'était pas un autre), peut-être parce que Platon ne l'a jamais nommé. Or de tous les sophistes, il est peut-être le plus grand » (p. 115). Plusieurs interprètes, dont Heidegger, avaient souligné l'importance de la conception antiphonienne de la nature comme arrythmiston (" libre de structure "). G. Romeyer-Dherbey appuie sur cette ontologie de l'in-forme, une philosophie existentielle de l'irréversible, attachante et saisissante à la fois. Il est aussi fait une juste place à l'activité onirocritique et psychiatrique d'Antiphon.

Voilà donc un excellent livre.

Pierre Pellegrin. 
Eudaldo Forment GiRAlT, Introducción a la metafísica. Barcelona, Ed. Universitat de Barcelona, 1984. 16,7 × 23,7, 202 p., bibliogr.

L'auteur, docteur en philosophie, enseigne la métaphysique à l'Université de Barcelone depuis 1971 et est l'auteur de multiples travaux (entre autres, Ser y persona, Persona y modo substancial); il appartient à l'École thomiste de Barcelone, fondée par R. Orlandis, pénétrée d'augustinisme avec J. Bofill (mort en 1965) et continuée par Francisco Canals Vidal (dont les Cuestiones de fundamentación inspirent Forment). Son nouvel ouvrage cherche à définir l'essence de la métaphysique, comme une science de l'être et comme un savoir absolu ; avec Heidegger, il déplore "l'oubli de l'être ", depuis deux siècles ; l'erreur de Kant serait d'avoir confondu la métaphysique traditionnelle et classique - dogmatique -, qui dérivait de la contestable doctrine de l'intuition eidétique du connaître, avec la métaphysique aristotélico-thomiste, tout à fait étrangère à une telle doctrine. Après un court, mais incisif, prologue, l'introduction étudie (notamment, à travers Balmes) « la constitution de la métaphysique "; le chapitre I est consacré à ses trois "fondements " (la connaissance propre de l'esprit, le principe de non-contradiction, l'affirmation de l'existence de l'être et de la vérité) ; le chapitre II scrute "l'objet de la métaphysique " (à l'aide de Sylvestre de Ferrare et de Cajetan, puis de Suárez) ; le chapitre III porte sur "l'analogie " (en s'appuyant sur Santiago María Ramírez, Cajetan, Suárez, etc.) et reconnaît l'écart entre la nature de Dieu et nos concepts ; le chapitre IV compare l'étant et l'être, à la lumière de Bál ez et de Cajetan, en concevant l'être comme acte ; l'épilogue s'efforce de démontrer comment « l'unilatéralisme essentialiste " (p. 177) laisse de côté la dimension existentielle et, donc, l'existence nécessaire de Dieu. « La métaphysique est une science unique et parler de métaphysiques spéciales n'a aucun sens [...] ; étant finalement théologie, la métaphysique peut être considérée comme la principale des sciences, car c'est par elle seulement que l'on peut acquérir quelques lueurs sur Dieu » (p. 184). Même si les convictions d'E. Forment ne rallient pas tous ses lecteurs, sa méthode rigoureuse mérite notre respect et notre estime. Sa bibliographie est éclectique (d'Alcorta, d'Alquié et du R.P. Pierre C. Courtès, à C. Fabro, Forest, Funke, Heidegger, Ricœur, Verneaux, J. Wahl ou Xirau).

Alain GuY.

Eudaldo Forment Giralt, Fenomenologia descriptiva del lenguaje. Segunda edicion. Barcelona, Promociones Publicaciones Universitarias, 1984. $12,5 \times 19,352$ p., bibliogr.

Réédition littérale et très attendue de la première édition (1981), depuis longtemps épuisée, cet ouvrage savant et brillant se propose de prouver que la linguistique actuelle se fonde sur la phénoménologie du langage ; à cette fin, 
E. Forment Giralt part d'une analyse approfondie des Recherches logiques (1901) de Husserl, pour élargir ensuite ses perspectives. Le volume se compose de deux parties. Les quatre premiers chapitres étudient l'expression : son concept, sa constitution, son contenu significatif et son contenu objectif ; les deux derniers chapitres exposent la " grammaire pure » ou " générale » (c'est-à-dire une science qui étudie l'a priori de l'expression) et en cherchent la portée comme fondement possible de la linguistique. $\dot{A}$ cet effet, sont successivement scrutés le Cours de F. de Saussure, les présupposés de la grammaire traditionnelle et de la linguistique historique, le structuralisme linguistique européen (Cercle de Prague, avec Troubetzkoï et Cercle de Copenhague, avec Hjelmlev et Brondal), ou nord-américain (l'antimentalisme et le descriptivisme de Bloomfield), enfin, le post-structuralisme actuel de Chomsky.

Au passage, on note que Forment s'oppose à la conception de Rossi, qui confond les trois acceptions du concept de «Contenu». Les conclusions sont quadruples : la phénoménologie légitime pleinement les acquis de la linguistique du structuralisme classique; la grammaire pure fonde cette linguistique ; au contraire, le structuralisme antimentaliste s'y oppose ; enfin, à la lumière de l'étape présente de la linguistique, on s'aperçoit que la grammaire pure coïncide avec la grammaire transformationaliste. En résumé, « la recherche phénoménologique du langage fonde la grammaire générative, puisque, de la même façon que la description phénoménologique de l'expression fonde la grammaire pure, elle fonde aussi, étant donné la coïncidence entre les deux, la grammaire générative transformationnelle " (p. 336). Espérons qu'une traduction française de ce beau livre paraîtra bientôt !

Alain GuY.

\section{HISTOIRE DES SCIENCES}

Rémy Chauvin, La Biologie de l'esprit. Monaco, Ed. du Rocher, 1985. $15 \times 24$, 220 p., fig., bibliogr. (" L'Esprit et la matière ").

L'éthologie des insectes sociaux a pour éminent spécialiste le professeur en Sorbonne Rémy Chauvin. Il vient de publier La Biologie de l'esprit, ouvrage captivant sur l'Évolution animale entière. Il ne craint pas d'y réfuter son explication par Darwin : une « sélection » comme celle qui isole et améliore nos races domestiques, mais « naturelle » : le « milieu » triant, épanouissant les mutations favorables. Or les mutations sont particulièrement nombreuses (et exploitées expérimentalement) chez la mouche drosophile, et elle n'a pas changé depuis 50 millions d'années, où elle se fossilisait dans l'ombre ; il en va de même des bactéries et autres espèces stables (« panchroniques ", fossiles vivants) (p. 23). Notre élevage s'appuie aussi sur une consanguinité ; celle-ci est très rare, même dans les sociétés animales. 